\title{
HORSESHOE IN A CLASS OF PLANAR MAPPINGS
}

\author{
YAN HUANG AND XIAO-SONG YANG
}

Received 6 March 2005; Accepted 2 June 2005

Bursting dynamics of mappings is investigated in this paper. We first present stability analysis of the mappings' equilibria with various parameters. Then for three mappings $P$, $\bar{P}$, and $\widehat{P}$ with different parameters, we study their powers $P^{4}, \bar{P}^{6}$, and $\hat{P}^{4}$. We show that the mappings thus obtained are chaotic by giving a rigorous verification of existence of horseshoes in these mappings. Precisely, we prove that the mapping $\bar{P}^{6}$ is semiconjugate to the 3-shift mapping; the mappings $P^{4}$ and $\widehat{P}^{4}$ are semiconjugate to the 4-shift mapping.

Copyright (c) 2006 Y. Huang and X.-S. Yang. This is an open access article distributed under the Creative Commons Attribution License, which permits unrestricted use, distribution, and reproduction in any medium, provided the original work is properly cited.

\section{Introduction}

Bursting is ubiquitous in physical and biological systems, especially in neural systems [3]. Studying networks of bursters in neural systems poses challenging mathematical problems. Even simulating such networks is a computational challenge, since thousands of stiff nonlinear ordinary differential equations (ODEs) may be involved.

Bursting dynamics of mappings has recently been investigated by physicists $[1,5,6,2]$. Using a discrete-time system, say $x_{n+1}=f\left(x_{n}\right)$, instead of a system of ODEs, provides one with a number of theoretical and computational advantages. For example, it is possible to explore collective behavior of millions of coupled discrete-time bursters with only modest computational effort.

In order to study the phenomenon of bursting, a simple discrete-time model of spiking neurons was proposed in [3], which is of the following form:

$$
\begin{aligned}
& \text { I: } \begin{array}{l}
v_{n+1}=0.04 v_{n}^{2}+6 v_{n}+140-u_{n}+I, \\
u_{n+1}=0.004 v_{n}+0.98 u_{n},
\end{array} \\
& \text { if } v_{n} \geq 30 m V, \text { then }\left\{\begin{array}{l}
v_{n} \longleftarrow c, \\
u_{n+1} \longleftarrow u_{n}+d .
\end{array}\right.
\end{aligned}
$$

Hindawi Publishing Corporation

Discrete Dynamics in Nature and Society

Volume 2006, Article ID 67908, Pages 1-10

DOI 10.1155/DDNS/2006/67908 
In this paper, we study a more general mapping as follows.

Let $l=0.04, n=6, \bar{I}=41$, (1.1) can be rewritten as follows:

$$
\begin{aligned}
& \text { II: } \begin{array}{l}
v_{n+1}=0.04 v_{n}^{2}+6 v_{n}+41-u_{n}, \\
u_{n+1}=a v_{n}+b u_{n},
\end{array} \\
& \text { if } v_{n} \geq 30 m V, \quad \text { then }\left\{\begin{array}{l}
v_{n} \longleftarrow c, \\
u_{n+1} \longleftarrow u_{n}+d .
\end{array}\right.
\end{aligned}
$$

Depending on the values of the parameters, this mapping can produce a variety of bursting patterns [3]. In addition, this simple model can reproduce 20 most fundamental neuron-computational properties of biological spiking neurons summarized in [3].

\section{The stability of equilibria with various parameters}

We first study in this section the stability of equilibria of (1.2) for different parameters.

Equation (1.2) has two equilibria, let $A$ and $B$ denote them. Let the modules of the eigenvalues of $A$ be $m_{11}$ and $m_{12}$, the modules of the eigenvalues of $B$ be $m_{21}$ and $m_{22}$. $m_{11}, m_{12}, m_{21}$, and $m_{22}$ vary with the parameters $a$ and $b$ as shown in Figure 2.1, $m_{i, j}=$ $m_{i, j}(a, b), i, j=1,2$. Denote by $M$ the plane $z=1$ in Figure 2.1. With the reference plane $M$, it can be concluded that $A$ is a saddle point when $a$ and $b$ vary from 0 to 0.99 , respectively, and $B$ may be a saddle point, a stable equilibrium, or an unstable equilibrium when $a$ and $b$ varies from 0 to 0.99 .

Typical values for $c$ and $d$ are $c=-56, d=-16$. When $a=0.24$ and $b=0.6$, let $P$ denote the mapping II. The mapping $P$ has two saddle points $(-10.2786,-6.1672)$ and $(-99.7214,-59.8328)$. When $a=0.64$ and $b=0.2$, let $\bar{P}$ denote the mapping II, the mapping $\bar{P}$ has a saddle point $(-10.8917,-8.7134)$ and a stable equilibrium $(-94.1083$, $-75.2866)$.

In the next section, we recall a result on horseshoes theory developed in $[8,9]$, which is essential for rigorous verification of existence of chaos in the systems discussed in this paper.

\section{Review of a topological horseshoe theorem}

Let $X$ be a metric space, $D$ is a compact subset of $X$, and $f: D \rightarrow X$ is mapping satisfying the assumption that there exist $m$ mutually disjoint subsets $D_{1}, \ldots$, and $D_{m}$ of $D$, the restriction of $f$ to each $D_{i}$, that is, $f \mid D_{i}$ is continuous.

Definition 3.1. Let $\gamma$ be a compact subset of $D$, such that for each $1 \leq i \leq m, \gamma_{i}=\gamma \cap D_{i}$ is nonempty and compact, then $\gamma$ is called a connection with respect to $D_{1}, \ldots, D_{m-1}$ and $D_{m}$.

Let $F$ be a family of connections $\gamma$ 's with respect to $D_{1}, \ldots, D_{m-1}$ and $D_{m}$ satisfying the following property:

$$
\gamma \in F \Longrightarrow f\left(\gamma_{i}\right) \in F
$$

Then $F$ is said to be an $f$-connected family with respect to $D_{1}, \ldots, D_{m-1}$ and $D_{m}$. 


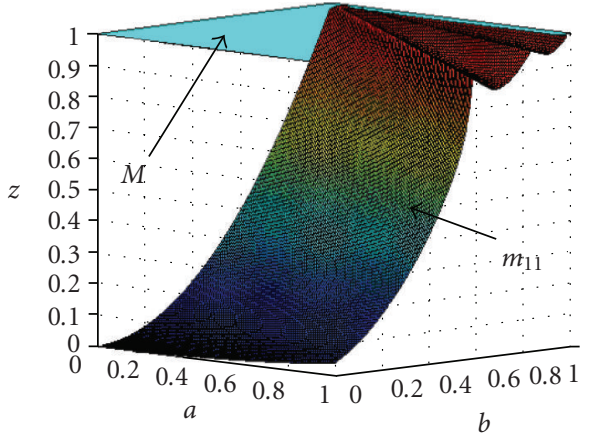

(a) $m_{11}$ as a function of the parameters $a$ and $b$.

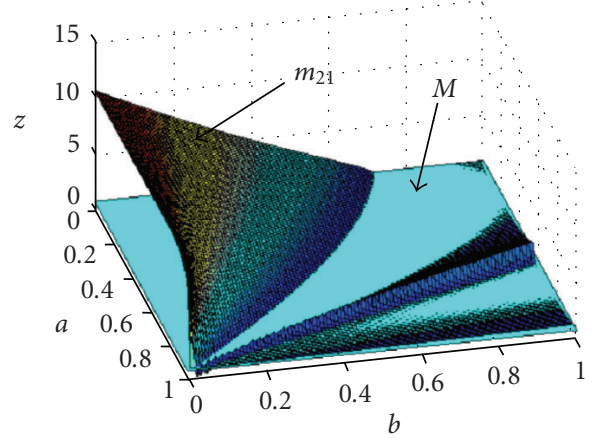

(c) $m_{21}$ as a function of the parameters $a$ and $b$.

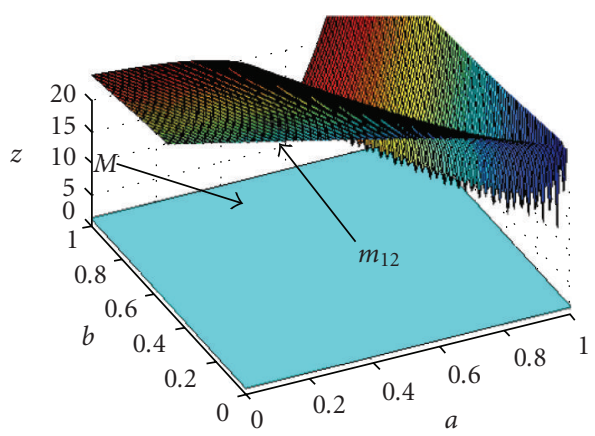

(b) $m_{12}$ as a function of the parameters $a$ and $b$.

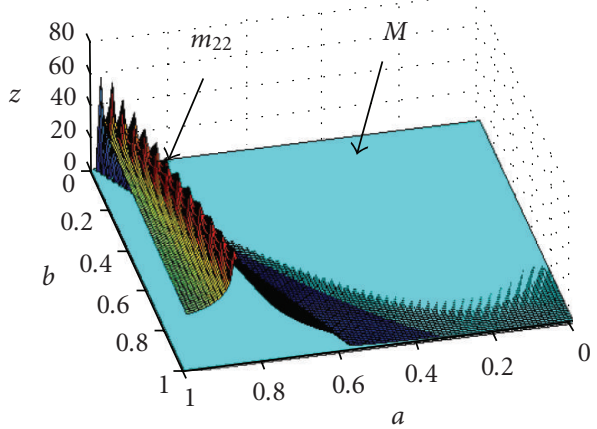

(d) $m_{22}$ as a function of the parameters $a$ and $b$.

Figure 2.1. The modules of the eigenvalues.

Theorem 3.2. Suppose that there exists an $f$-connected family $F$ with respect to $D_{1}, \ldots$, $D_{m-1}$ and $D_{m}$. Then there exists a compact invariant set $K \subset D$, such that $f \mid K$ is semiconjugate to $m$-shift

For the proof of this theorem, see [9].

Here the "semiconjugate to the $m$-shift" is conventionally defined in the following sense. If there exists a continuous and onto mapping

$$
h: K \longrightarrow \sum_{m}
$$

such that $h \circ f=\sigma \circ h$, then $f$ is said to be semiconjugate to $\sigma$, where $\sigma$ is the $m$-shift (mapping) and $\sum_{m}$ is the space of symbolic sequences to be defined below. Let $S_{m}=$ $\{1, \ldots, m\}$ be the set of nonnegative successive integer from 1 to $m$. Let $\sum_{m}$ be the collection of all one-infinite sequences with their elements of $S_{m}$, that is, every element $s$ of $\sum_{m}$ 
is of the following form:

$$
s=\left\{s_{1}, \ldots s_{m}, \ldots\right\}, \quad s_{i} \in S_{m} .
$$

Now consider another sequence $\bar{s}_{i} \in S_{m}$.

The distance between $s$ and $\bar{s}$ is defined as

$$
d(s, \bar{s})=\sum_{i=1}^{+\infty} \frac{1}{2^{|i|}} \frac{\left|s_{i}-\bar{s}_{i}\right|}{\left|s_{i}-\bar{s}_{i}\right|+1}
$$

with the distance defined as (3.4), $\sum_{m}$ is a metric space, and the $m$-shift mapping $\sigma$ : $\sum_{m} \rightarrow \sum_{m}$ is defined as follows [7]:

$$
\sigma(s)_{i}=s_{i+1}, \quad s=\left\{s_{1}, \ldots s_{m}, \ldots\right\} .
$$

For the concept of topological entropy, the reader can refer to [4]. We just recall the result stated in Lemma 3.3, which will be used in this paper.

Lemma 3.3. Let $X$ be a compact metric space, and $f: X \rightarrow X$ a continuous mapping. If there exists an invariant set $\Lambda \subset X$ such that $f \mid \Lambda$ is semiconjugate to the $m$-shift $\sigma$, then

$$
h(f) \geq h(\sigma)=\log m,
$$

where $h(f)$ denotes the entropy of the mapping $f$. In addition, for every positive integer $k$,

$$
h\left(f^{k}\right)=k h(f) .
$$

A well-known fact is that if the entropy of continuous mapping is positive, then the mapping is chaotic [4].

\section{Horseshoes for the mappings}

4.1. Horseshoes for the iteration of the mapping $p$ with two saddle points. Let $P$ denote the mapping corresponding to the parameters $a=0.24$ and $b=0.60, P$ has two saddle points. With these parameters, we find a quadrangle $D$ with its vertexes: $(-114.2947$, $-48.3442),(-41.7506,-55.1067),(-46.2846,63.6224)$ and $(-111.7742,-61.1316)$ by careful computation. Figure 4.1 is the quadrangle $D$ and its image under the fourth iteration of the mapping $P$ (that is $P^{4}$ ). It can be seen from Figure 4.1 that there exist discontinuous points. The resetting (1.3) is responsible for this phenomenon, because not all of the points on the edge of $D$ are reset at the same time.

Four disjoint quadrangles as defined in Definition 3.1 can been constructed, they are $d_{1}, d_{2}, d_{3}, d_{4}$. The vertexes' coordinates of $d_{1}, d_{2}, d_{3}, d_{4}$ are listed in Table 4.1. Their images under the mapping $P^{4}$ are shown in Figure 4.4.

In Figure 4.2, $d l_{i}$, are the left sides of $d_{i}, d r_{i}$ are the right sides of $d_{i}, i=1,2,3,4 . P^{4}\left(d l_{i}\right)$ and $P^{4}\left(d r_{j}\right)$ lie on the left side of $d l_{1}, i=1,3, j=2,4 ; P^{4}\left(d l_{i}\right)$ and $P^{4}\left(d r_{j}\right)$ lie on the right side of $d r_{4}, i=2,4, j=1,3$.

It is easy to see from Figure 4.2 that every line $l$ lying in $D$ and connecting the side $d l_{1}$ and $d r_{4}$ has nonempty connections with $d_{1}, d_{2}, d_{3}$ and $d_{4}$. Furthermore, $P^{4}\left(l \cap d_{1}\right)$ 


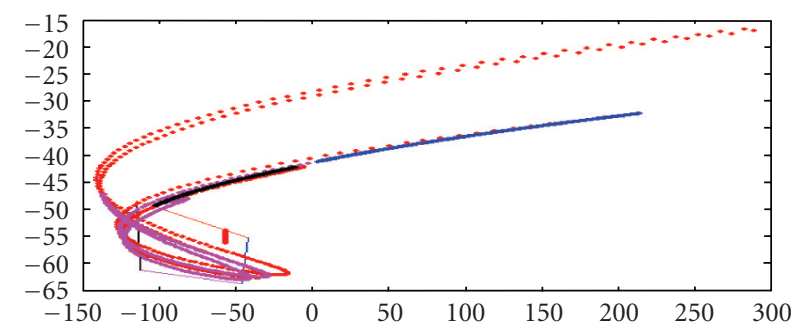

Figure 4.1. The quadrangle $D$ and its image under the mapping $P^{4}$.

Table 4.1. The vertexes' coordinates of $d_{1}, d_{2}, d_{3}, d_{4}$.

\begin{tabular}{llllll}
\hline$d_{1}$ & $(-105.9500,-49.1220)$ & $(-99.7860,-49.6970)$ & $(-93.1100,-61.8410)$ & $(-102.2800,-61.4930)$ \\
$d_{2}$ & $(-97.9720,-49.8660)$ & $(-81.3220,-62.290)$ & $(-93.2570,-50.3050)$ & $(-92.1270,-61.8790)$ \\
$d_{3}$ & $(-60.6120,-53.3480)$ & $(-56.9850,-53.6870)$ & $(-61.3470,-63.050)$ & $(-70.1880,-62.7130)$ \\
$d_{4}$ & $(-53.3580,-54.0250)$ & $(-49.3680,-54.3970)$ & $(-51.8510,-63.4110)$ & $(-58.7280,-63.1490)$ \\
\hline
\end{tabular}

connects $d l_{1}$ and $d r_{4}$ from the above arguments, $P^{4}\left(l \cap d_{i}\right)$ also connects $d l_{1}$ and $d r_{4}$, $i=2,3,4$. Therefore, it is easy to see, in view of Definition 3.1, that there exists a $P^{4}$ family with respect to these four subsets $d_{1}, d_{2}, d_{3}$ and $d_{4}$ for the mapping $P^{4}$. It follows from Theorem 3.2 that there exists an invariant set $K_{1}$ of $D$, such that $P^{4}$ restricted to $K_{1}$ is semiconjugated to 4 -shift dynamics. Let $h\left(P^{4}\right)$ be the entropy of the mapping $P^{4}$, it can be concluded from Lemma 3.3 that $h\left(P^{4}\right) \geq h(\sigma)=\log 4$, consequently the entropy of the mapping $P$ is not less than $(1 / 2) \log 2$. From above facts, it can be concluded that the mapping $P^{4}$ is chaotic.

Because $K_{1}$ is an invariant set, every point in $K_{1}$ cannot skip out of the quadrangle $D$ under iteration of the mapping $P$. Then, the resetting has nothing to do with the points in $K_{1}$.

4.2. Horseshoes for the iteration of the mapping $\bar{P}$ with a saddle point and a stable equilibrium. Let $\bar{P}$ denote the mapping corresponding to the parameters $a=0.64$ and $b=0.2$, the mapping $\bar{P}$ has a saddle point and a stable equilibrium. With these parameters, we find a quadrangle $\bar{D}$ with its vertexes $(-81.4747,-72.2222),(-39.4470$, $-84.9708),(-41.2903,-91.8713)$, and $(-83.3180,-83.5673)$ by means of careful computation.

Figure 4.3 is the quadrangle $\bar{D}$ and its image under the sixth iteration of the mapping $\bar{P}$, that is $\bar{P}^{6}$. The image of $\bar{D}$ under $\bar{P}^{6}$ has discontinuous part because that not all of the points on the edge of $\bar{D}$ are reset at the same time. Three disjoint quadrangles as defined in Definition 3.1 can also been constructed, they are $h_{1}, h_{2}, h_{3}$. The vertexes' coordinates of $h_{1}, h_{2}, h_{3}$ are listed in Table 4.2.

In Figure 4.4, $h l_{i}$, are the left sides of $h_{i}, h r_{i}$ are the right sides of $h_{i}, i=1,2,3 \cdot \bar{P}^{6}\left(h l_{1}\right)$, $\bar{P}^{6}\left(h r_{2}\right)$ and $\bar{P}^{6}\left(h r_{3}\right)$ lie on the left side of $h l_{1}, \bar{P}^{6}\left(h r_{1}\right), \bar{P}^{6}\left(h l_{2}\right)$ and $\bar{P}^{6}\left(h l_{3}\right)$ lie on the right side of $h r_{3}$. 


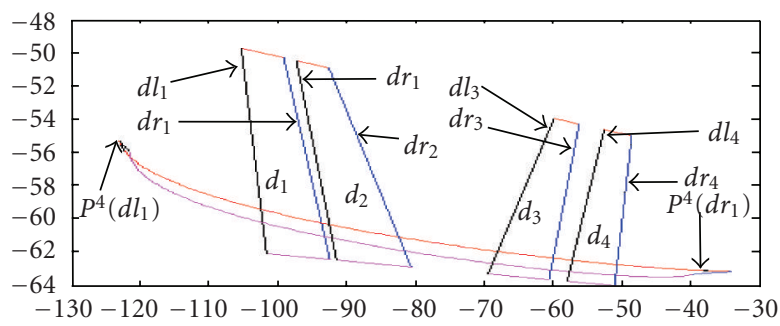

(a) The four disjoint subsets of $D$ and the image of $d_{1}$ under the mapping $P^{4}$.

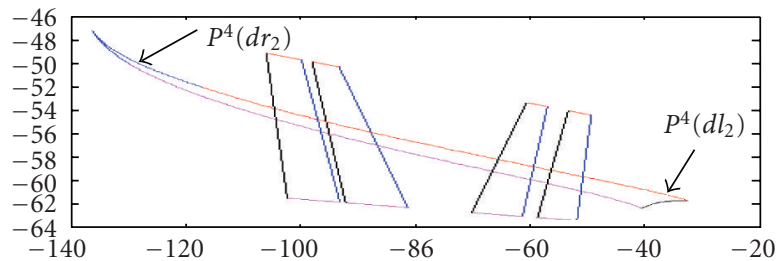

(b) The four disjoint subsets of $D$ and the image of $d_{2}$ under the mapping $P^{4}$.

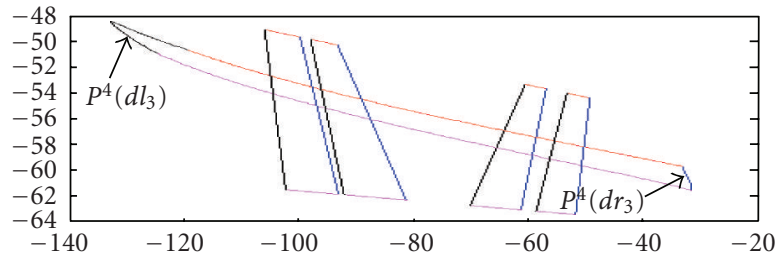

(c) The four disjoint subsets of $D$ and the image of $d_{3}$ under the mapping $P^{4}$.

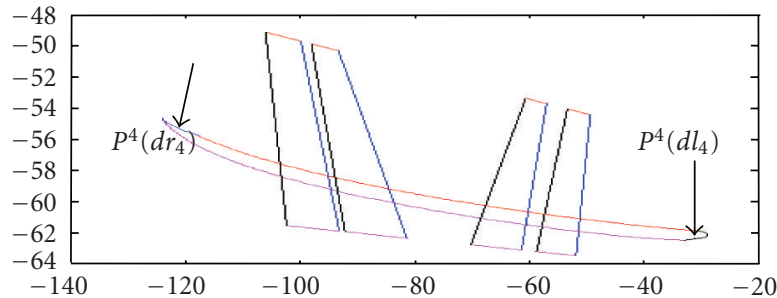

(d) The four disjoint subsets of $D$ and the image of $d_{4}$ under the mapping $P^{4}$.

Figure 4.2. The four disjoint subsets of $D$ and their images under the mapping $P^{4}$.

It is easy to see from Figure 4.4 that every line $l$ lying in $\bar{D}$ and connecting the side $h l_{1}$ and $h r_{3}$ has nonempty connections with $h_{1}, h_{2}$, and $h_{3}$. Furthermore, $\bar{P}^{6}\left(l \cap h_{i}\right)$ connects $h l_{1}$ and $h r_{3}$ from the above arguments, $i=1,2,3$. In view of Definition 3.1, there exists a $\bar{P}^{6}$-family with respect to these three subsets $h_{1}, h_{2}$, and $h_{3}$ for the mapping $\bar{P}^{6}$. It follows from Theorem 3.2 that there exists an invariant set $K_{2}$ of $\bar{D}$, such that $\bar{P}^{6}$ restricted to $K_{2}$ 


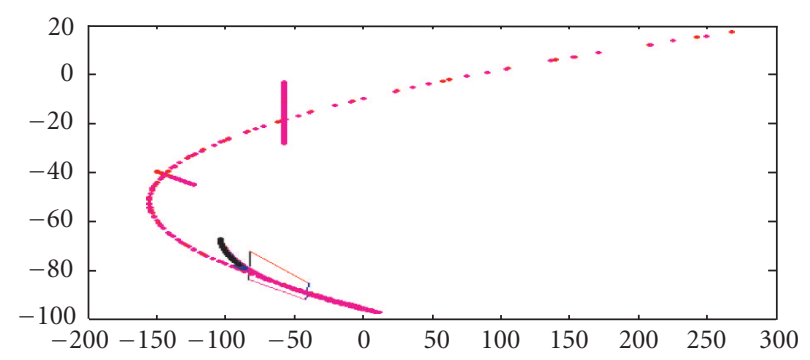

Figure 4.3. The quadrangle $\bar{D}$ and its image under the mapping $\bar{P}^{6}$.

Table 4.2. The vertexes' coordinates of $h_{1}, h_{2}, h_{3}$.

\begin{tabular}{llllll}
\hline$h_{1}$ & $(-61.9320,-78.1500)$ & $(-59.6200,-78.8510)$ & $(-65.2460,-87.1380)$ & $(-67.9780,-86.5980)$ \\
$h_{2}$ & $(-58.5700,-79.1700)$ & $(-62.0940,-87.7610)$ & $(-57.9390,-79.3610)$ & $(-62.7240,-87.6360)$ \\
$h_{3}$ & $(-41.3380,-84.3970)$ & $(-39.4470,-84.9708)$ & $(-41.2903,-91.8713)$ & $(-44.8630,-91.1650)$ \\
\hline
\end{tabular}

is semiconjugated to 3 -shift dynamics. Let $h\left(\bar{P}^{6}\right)$ be the entropy of the mapping $\bar{P}^{6}$, it can be concluded from Lemma 3.3 that $h\left(\bar{P}^{6}\right) \geq h(\sigma)=\log 3$, consequently the entropy of the mapping $\bar{P}$ is not less than (1/6) $\log 3$. The entropy of the mapping $\bar{P}$ is positive, then it can be concluded that the mapping $\bar{P}^{6}$ is chaotic.

Because $K_{2}$ is an invariant set, every point in $K_{2}$ cannot skip out of the quadrangle $\bar{D}$ under iteration of the mapping $\bar{P}$. Then, the resetting has nothing to do with the points in $K_{2}$.

4.3. Horseshoes for the iteration of the mapping I. The mapping I in [3] is a typical mapping with two saddle points. The mapping can produce a variety of bursting patterns, including those corresponding to IB (intrinsically bursting) and $\mathrm{CH}$ (chattering) neocortical neurons. A discussion on chaotic property in this mapping is given in this section.

Let $\hat{P}$ denote the mapping with $I=-99$. As the parameters in (1.1), the mapping $\hat{P}$ has two saddle points. With these parameters, we find a quadrangle $\hat{D}$ with its vertexes $(-47.902,-9.2532),(-28.4005,-9.2532),(-23.3627,-21.6558)$, and $(-53.424,-21.709)$ by means of careful computation.

Figure 4.5 is the image of the quadrangle $\hat{D}$ and its image under the fourth iteration of the mapping $\hat{P}$, that is $\hat{P}^{4}$. It can be seen from Figure 4.5 that there exist discontinuous points. The reason is the same as that in Section 4.1: the resetting (1.3) is responsible for this phenomenon. Four disjoint quadrangles as defined in Definition 3.1 can also been constructed, they are $k_{1}, k_{2}, k_{3}, k_{4}$. The vertexes' coordinates of $k_{1}, k_{2}, k_{3}, k_{4}$ are listed in Table 4.3. Their images under the fourth iteration of the mapping $\widehat{P}$ are shown in Figure 4.6.

In Figure 4.6, the four quadrangles are $k_{1}, k_{2}, k_{3}, k_{4}$ from left to right in turn. $k l_{i}$ is the left side of $k_{i}, k r_{i}$ is the right side of $k_{i}, i=1,2,3,4 . \widehat{P}^{4}\left(k l_{i}\right)$ and $\hat{P}^{4}\left(k r_{j}\right)$ lie on the left side of $k l_{1}, i=2,4, j=1,3 ; \hat{P}^{4}\left(k l_{i}\right)$ and $\hat{P}^{4}\left(k r_{j}\right)$ lie on the right side of $k r_{4}, i=1,3, j=2,4$. 


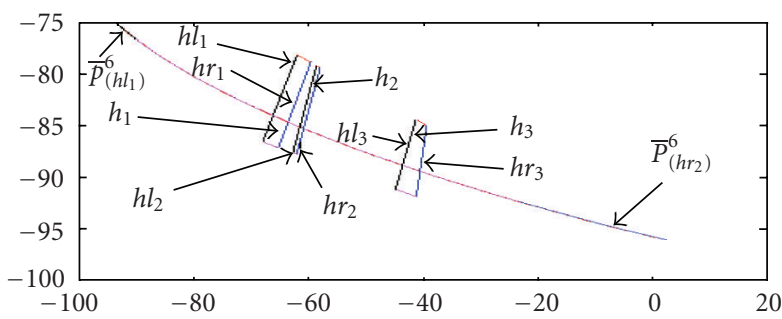

(a) The three disjoint subsets of $\bar{D}$ and the image of $h_{1}$ under the mapping $\bar{P}^{6}$.

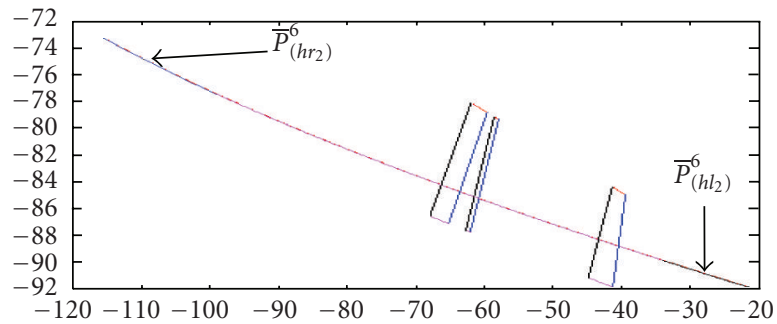

(b) The three disjoint subsets of $\bar{D}$ and the image of $h_{2}$ under the mapping $\bar{P}^{6}$.

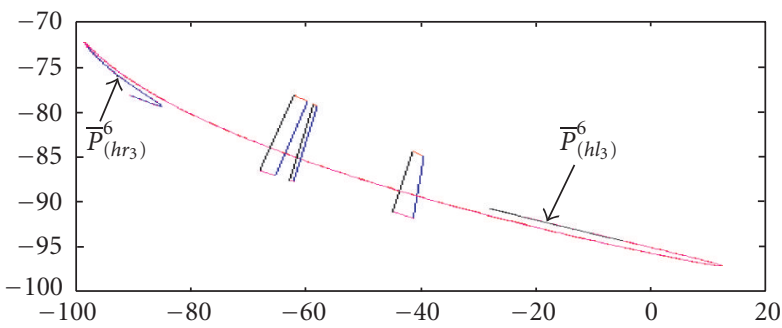

(c) The three disjoint subsets of $\bar{D}$ and the image of $h_{3}$ under the mapping $\bar{P}^{6}$.

Figure 4.4. The three disjoint subsets of $\bar{D}$ and their image under the mapping $\bar{P}^{6}$.

Table 4.3. The vertexes' coordinates of $k_{1}, k_{2}, k_{3}, k_{4}$.

\begin{tabular}{lllll}
\hline$k_{1}$ & $(-45.172,-9.2532)$ & $(-44.879,-9.2532)$ & $(-48.915,-21.701)$ & $(-49.366,-21.702)$ \\
$k_{2}$ & $(-42.734,-9.2532)$ & $(-42.149,-9.2532)$ & $(-45.608,-21.695)$ & $(-46.51,-21.697)$ \\
$k_{3}$ & $(-37.079,-9.2532)$ & $(-36.396,-9.2532)$ & $(-39.596,-21.685)$ & $(-40.648,-21.686)$ \\
$k_{4}$ & $(-33.568,-9.2532)$ & $(-33.178,-9.2532)$ & $(-35.688,-21.678)$ & $(-36.289,-21.679)$ \\
\hline
\end{tabular}

With the same analysis of the fourth paragraph in Sections 4.1 and 4.2, it can be concluded that there exists a $\hat{P}^{4}$-family with respect to these four subsets $d_{1}, d_{2}, d_{3}$ and $d_{4}$ for the mapping $\hat{P}^{4}$, and an invariant set $K_{3}$ of $\hat{D}$, such that $\hat{P}^{4}$ restricted to $K_{3}$ is 


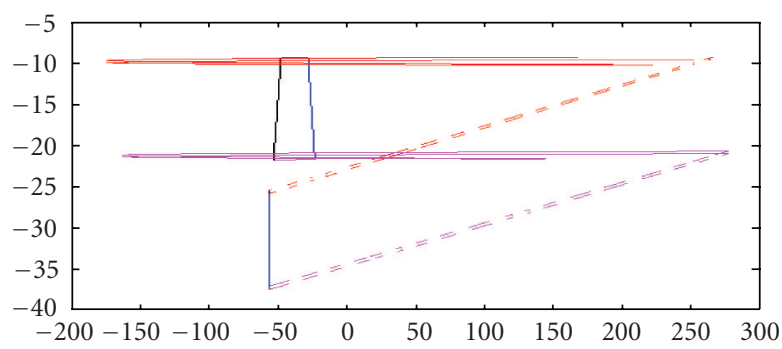

Figure 4.5. The quadrangle $\hat{D}$ and its image under the mapping $\hat{P}^{4}$.

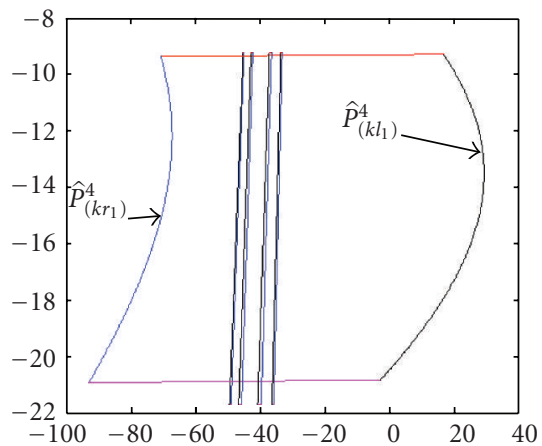

(a) The image of $k_{1}$ under the mapping $\widehat{P}^{4}$.

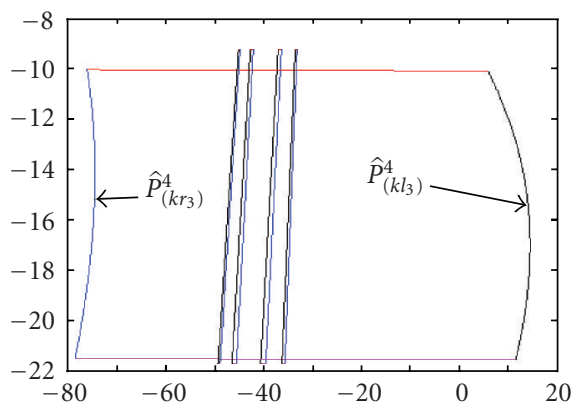

(c) The image of $k_{3}$ under the mapping $\widehat{P}^{4}$.

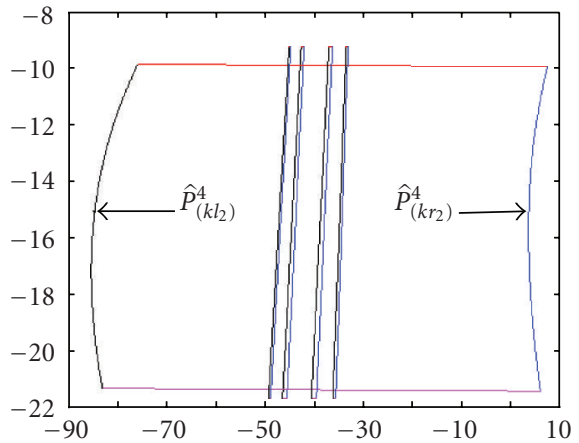

(b) The image of $k_{2}$ under the mapping $\widehat{P}^{4}$.

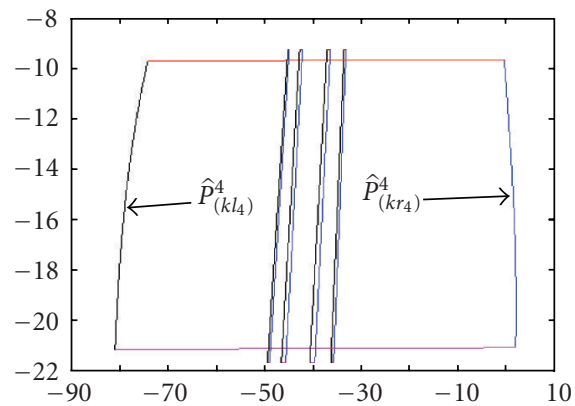

(d) The image of $k_{4}$ under the mapping $\hat{P}^{4}$.

Figure 4.6. The four disjoint subsets of $\widehat{D}$ and their images under the mapping $\widehat{P}^{4}$.

semiconjugated to 4 -shift dynamics. Let $h\left(\hat{P}^{4}\right)$ be the entropy of the mapping $\hat{P}^{4}$, it can be concluded from Lemma 3.3 that $h\left(\hat{P}^{4}\right) \geq h(\sigma)=\log 4$, consequently the entropy of the mapping $\hat{P}$ is not less than $(1 / 2) \log 2$. From above facts, it can be concluded that the mapping $\widehat{P}^{4}$ is chaotic. 
Because $K_{3}$ is an invariant set, every point in $K_{3}$ cannot skip out of the quadrangle $\hat{D}$ under iteration of the mapping $\hat{P}$. Then, the resetting has nothing to do with the points in $K_{3}$.

\section{Conclusion}

Studying networks of bursters in neural systems poses challenging mathematical problems. A bursting dynamics of mappings has been investigated in this paper. Based on the stability analysis of the system's equilibria with various parameters, we study three mappings of the system, that is $P^{4}, \bar{P}^{6}$ and $\hat{P}^{4}$. We show that the mappings are chaotic by giving a rigorous verification for existence of horseshoes in these mappings. We prove that the mapping $\bar{P}^{6}$ is semiconjugate to the 3 -shift mapping, the mapping $P^{4}$ and $\widehat{P}^{4}$ are semiconjugate to the 4 -shift mapping. Then, the mappings $P^{4}, \bar{P}^{6}$, and $\widehat{P}^{4}$ are chaotic.

\section{Acknowledgments}

The research is partially supported by Talents Foundation of Huazhong University of Science and Technology (0101011092), and partially supported by Program for New Century Excellent Talents in University. The first author is supported by Foundation for Excellent Ph.D. Thesis of Huazhong University of Science and Technology.

\section{References}

[1] B. Cazelles, M. Courbage, and M. Rabinovich, Anti-phase regularization of coupled chaotic maps modelling bursting neurons, Europhysics Letters 56 (2001), no. 4, 504-509.

[2] G. de Vries, Bursting as an emergent phenomenon in coupled chaotic mappings, Physical Review E. Statistical, Nonlinear, and Soft Matter Physics 64 (2001), no. 5, 051914.

[3] E. M. Izhikevich and F. Hoppensteadt, Classification of bursting mappings, International Journal of Bifurcation and Chaos in Applied Sciences and Engineering 14 (2004), no. 11, 3847-3854.

[4] C. Robinson, Dynamical Systems. Stability, Symbolic Dynamics, and Chaos, Studies in Advanced Mathematics, CRC Press, Florida, 1995.

[5] N. F. Rulkov, Regularization of synchronized chaotic bursts, Physical Review Letters 86 (2001), no. $1,183-186$.

[6] _ Modeling of spiking-bursting neural behavior using two-dimensional map, Physical Review E. Statistical, Nonlinear, and Soft Matter Physics 65 (2002), no. 4, 041922, 9.

[7] S. Wiggins, Introduction to Applied Nonlinear Dynamical Systems and Chaos, Texts in Applied Mathematics, vol. 2, Springer, New York, 1990.

[8] X.-S. Yang, Metric horseshoes, Chaos Solitons Fractals 20 (2004), no. 5, 1149-1156.

[9] X.-S. Yang and Y. Tang, Horseshoes in piecewise continuous maps, Chaos Solitons Fractals 19 (2004), no. 4, 841-845.

Yan Huang: Department of Mathematics, Huazhong University of Science and Technology, Wuhan 430074, China

Current address: Department of Control Science and Engineering, Huazhong University of Science and Technology, Wuhan 430074, China

E-mail address: huangyan2323@163.com

Xiao-Song Yang: Department of Mathematics, Huazhong University of Science and Technology,

Wuhan 430074, China

E-mail address: yangxs@cqupt.edu.cn 


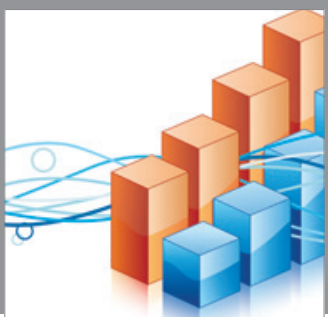

Advances in

Operations Research

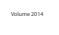

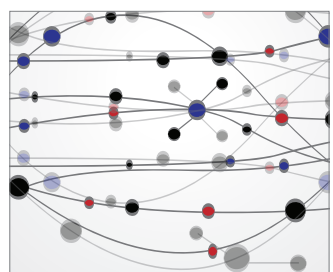

\section{The Scientific} World Journal
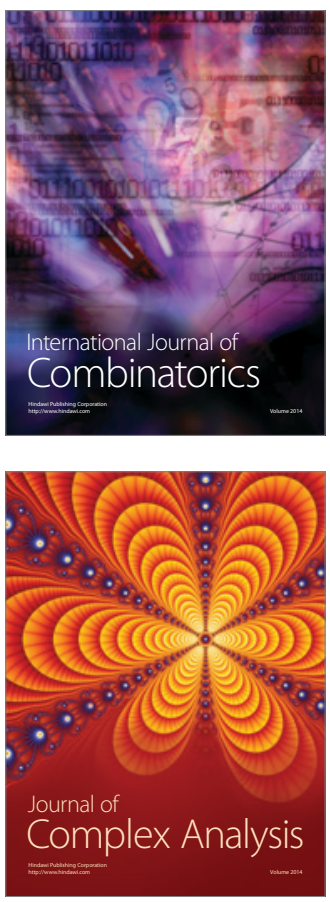

International Journal of

Mathematics and

Mathematical

Sciences
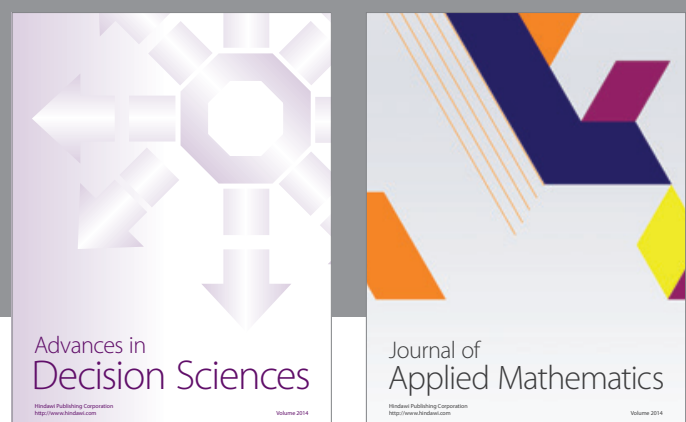

Journal of

Applied Mathematics
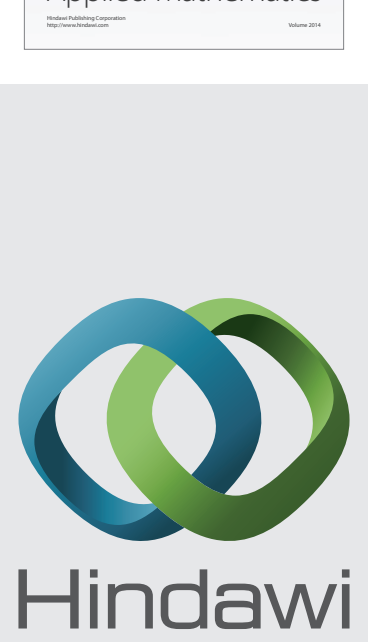

Submit your manuscripts at http://www.hindawi.com
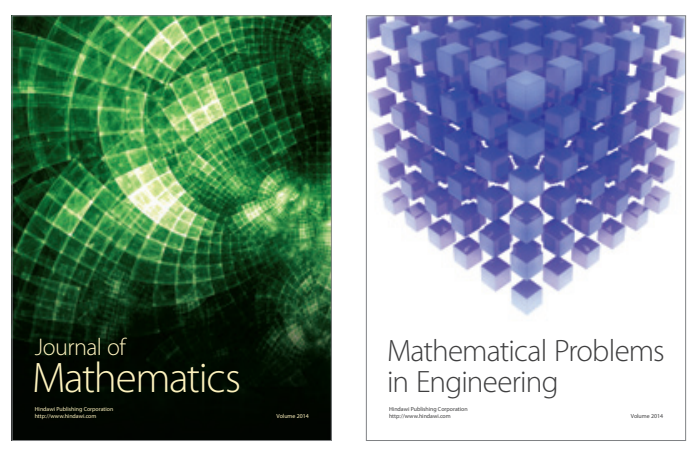

Mathematical Problems in Engineering
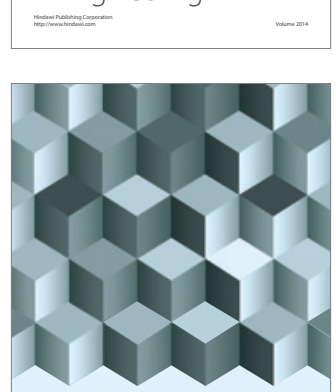

Journal of

Function Spaces
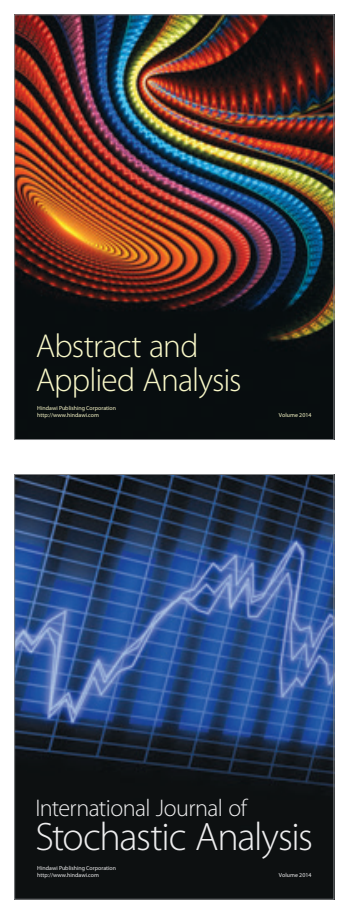

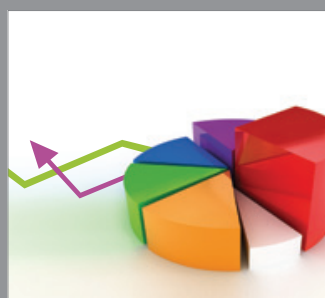

ournal of

Probability and Statistics

Promensencen
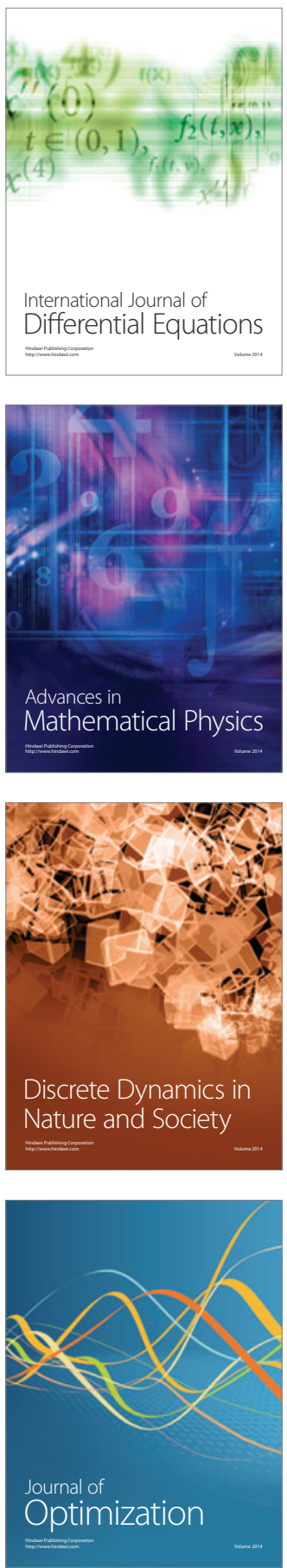